\title{
Microwave and Vacuum Assisted Fixation and Cytochemical Localization for Lungs of Chicken, Gallus domesticus
}

\author{
E. Ann Ellis
}

Biological Electron Microscopist, P. O. Box 6124, Thomasville, GA 31758, USA

Proper fixation of lung tissue for morphological and cytochemical studies by transmission electron microscopy (TEM) is difficult by conventional (perfusion and immersion) fixation processes. A broiler model (Gallus domesticus) of pulmonary hypertension was the source of lungs for this study. Microwave and intermittent vacuum were used in order to have better structural and enzymatic preservation. Bench cytochemical localization methods were modified for microwave use in terms of time and wattage. This paper presents the details of specimen preparation that were previously published to document oxidative stress by NADH oxidase (NOX) [1] in lungs of broilers maintained under hypoxia for four weeks.

Lungs were perfused through the left ventricle of the heart with heparinized saline followed by $10-15 \mathrm{ml}$ of $1 \%$ glutaraldehyde (v/v)-4\% paraformaldehyde (w/v) in $0.1 \mathrm{M}$ HEPES buffer, $\mathrm{pH} 7.4$ at $37^{\circ} \mathrm{C}$. Lungs were carefully removed by blunt dissection and placed in 10 volumes of cold $\left(5^{\circ} \mathrm{C}\right)$ fixative as above for approximately $1 \mathrm{hr}$. The fixative was replaced with cold 5\% acrolein (v/v), $0.1 \mathrm{M} \mathrm{HEPES} \mathrm{buffer,} \mathrm{pH}$ 7.4 and the lungs in $100 \mathrm{ml}$ tripour plastic beakers were placed in crushed ice in the vacuum chamber of a Pelco Biowave (Ted Pella, Inc., Redding CA). All fixation and wash cycles were done at $5{ }^{\circ} \mathrm{C}, 250$ watts power with intermittent vacuum (ON $30 \mathrm{sec}$; vacuum OFF $30 \mathrm{sec}$ ). Lungs were microwaved on a 6 minute cycle ( 2 min ON; 2 min OFF; 2 min ON). Fixative was removed and replaced with buffer wash consisting of 0.15 M HEPES buffer, $\mathrm{pH} 7.4$ plus 5\% sucrose (w/v) and 1\% dimethyl sulfoxide (v/v). Three 1 minute microwave cycles were used for washing at $1 \mathrm{~min}$ at 250 watts with intermittent vacuum. Lungs were stored in the cold in buffer wash until they were dissected into small pieces for reaction with $\mathrm{NADH}$ oxidase to determine localization of areas of oxidative stress [2]. The microwave was equilibrated to $37^{\circ} \mathrm{C}$ and samples were allowed to come to room temperature in new buffer wash plus $0.1 \mathrm{M}$ glycine to remove any unreacted aldehydes. Samples were then incubated in a freshly prepared preincubation mixture of cerium chloride, aminotriazole, and $0.0002 \%$ Triton X-100 (v/v) in $0.1 \mathrm{M}$ Tris-maleate buffer, $\mathrm{pH}$ plus $7 \%$ sucrose. The samples were reacted for two 6 min cycles $[2 \mathrm{~min}$ $\mathrm{ON} ; 2 \mathrm{~min} \mathrm{OFF} ; 2 \mathrm{~min} \mathrm{ON}$ ] at 250 watts and $37^{\circ} \mathrm{C}$. This is the equivalent of incubating for 30 minutes in a $37^{\circ} \mathrm{C}$ shaking water bath. The reaction mixture was then changed to a complete freshly prepared reaction mixture consisting of the substrate $\mathrm{NADH}$, the chromagen cerium chloride, an inhibitor of catalse and glutathione peroxidase (aminotriazole) and $0.0002 \%$ Triton X-100 (v/v). This was incubated in the microwave for a $6 \mathrm{~min}$ cycle, as above at 250 watts. Three additional changes of complete reaction mixture and microwaving at 250 watts at 6 min cycles were done. These four 6 min cycles are the equivalent of two 30 minute changes in a $37{ }^{\circ} \mathrm{C}$ shaking water bath. Samples were removed from the microwave and washed on the bench in two cold washes of 0.1 M HEPES buffer, $\mathrm{pH} \mathrm{7.4.} \mathrm{followed} \mathrm{by}$ osmication overnight in 1\% osmium tetroxide, 0.1 M HEPES buffer, $\mathrm{pH} 7.4$ in the cold. Specimens are then dehydrated, infiltrated with epoxy resin [3] and embedded. Gold sections were cut and examined in the TEM without post staining. Grids were examined and photographed in a JEOL 1200EX TEM at an accelerating voltage of $100 \mathrm{kV}$. NADH oxidase (NOX) localization results in an electron dense reaction product, cerium perhydroxide (Fig. 1). 
Microwave specimen preparation has been embraced because of the potential for rapid turnaround. However, in this work the microwave plus intermittent vacuum gave improved structural and enzymatic preservation such that a number of birds could be handled in a reproducible manner. This paper provides the details for better fixation and preparation of adult broiler lung for TEM and for cytochemical localization by using microwave and intermittent vacuum assisted techniques. The most important aspect is that 250 watts power and $37^{\circ} \mathrm{C}$ with a properly calibrated microwave and intermittent vacuum yields the same or better results as a 15 minute cycle in a $37^{\circ} \mathrm{C}$ shaking water bath. Changing to fresh reaction medium at each step is also important. The microwave parameters presented above can now be applied to conventional bench methods.

[1] J. Bautista-Ortega et al, Microscopy and Microanalysis (2010), p. 964.

[2] E. A. Ellis and M. B. Grant in "Oxidants and Antioxidants" ed. D Armstrong, (Humana Press, Totowa, New Jersey, p.3.

[3] E. A. Ellis, Microscopy Today (2006)14(4), p. 32.

[4] Supported by Hatch Project H70280.

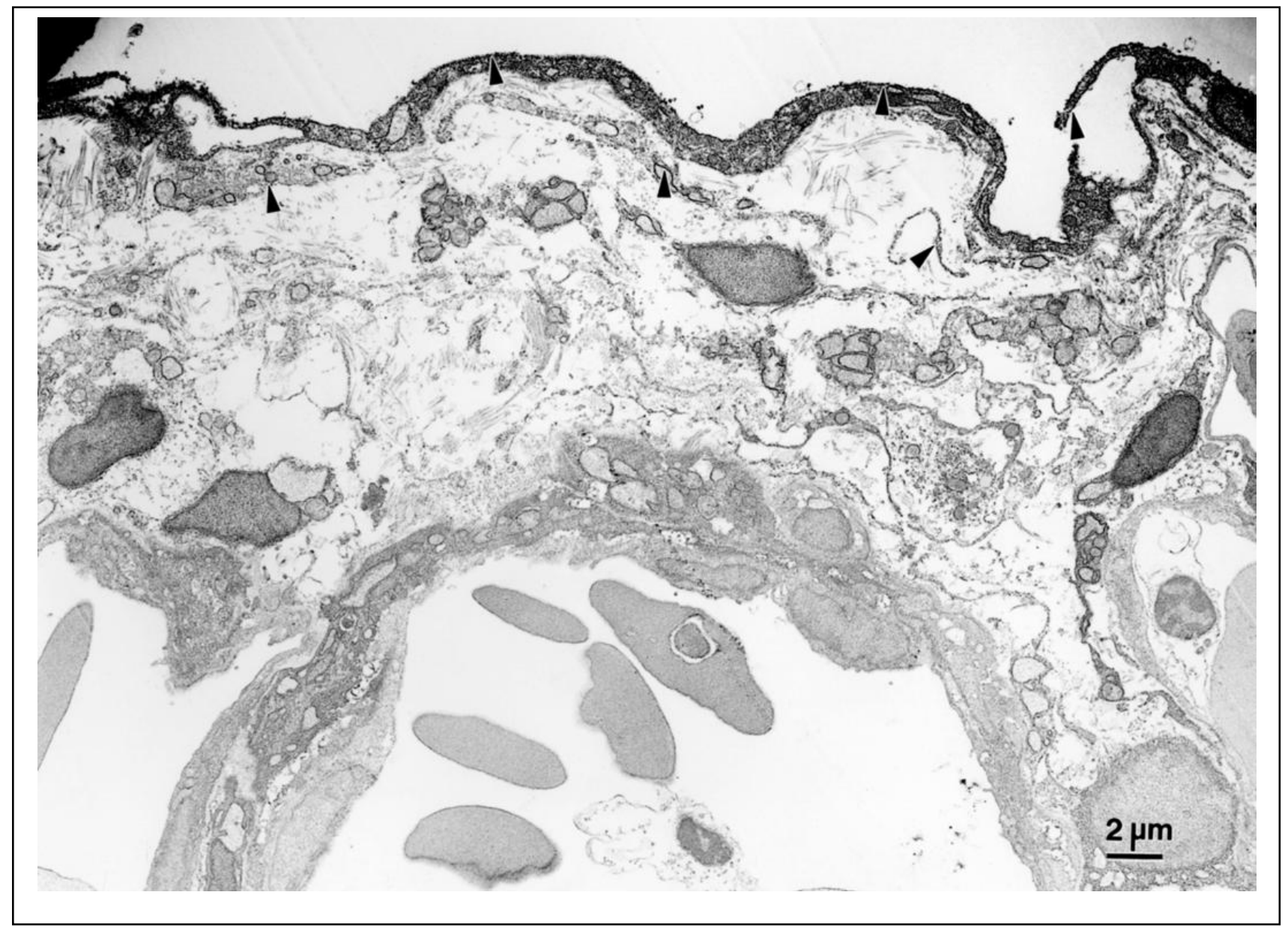

Figure 1. Localization of oxidative stress (NADH oxidase (NOX)) by an electron dense precipitate, cerium perhydroxide (arrow heads) in lung of a broiler maintained under hypobaric hypoxia for four weeks. There was heavy localization in the parabronchiolar epithelium and also low but consistent localization in the vascular endothelial cells and on the red blood cells. 